\title{
Discrimination économique de méthodes de traitements de sédiments marins contaminés à l'aide de liants hydrauliques
}

\author{
Laurent SANNIER $^{1,2}$, Daniel LEVACHER ${ }^{1}$, Mickaël JOURDAN ${ }^{2}$ \\ ${ }^{1}$ Université de Caen, Faculté des Sciences, UMR 6143 CNRS - M2C, \\ Esplanade de la Paix, 14032 Caen, France \\ hugomar.sannier@numericable.com ; daniel.levacher@unicaen.fr \\ ${ }^{2}$ Conseil Général du Var, Direction des Ports / Division Travaux neufs \\ 390, avenue des Lices - 83076 TOULON Cedex, France \\ lsannier@cg83.fr;mjourdan@cg83.fr
}

\section{Résumé :}

Pour une meilleure gestion à terre des sédiments contaminés, considérés comme déchets, une unité pilote de traitements a été mise en œuvre sur un site expérimental proche de Toulon. Un large éventail de sédiments portuaires représentatif de la variabilité de ces déchets ont été acheminés sur cette plateforme pour y être traités et valorisés et ce, de manière opérationnelle dans le cadre du projet SEDIMARD 83 (GROSDEMANGE et al, 2008). Un des objectifs du projet a concerné la valorisation des sédiments traités par liant hydraulique en menant des essais de faisabilité de stabilisation/solidification sur les sédiments pré-traités et/ou traités tant du point de vue mécanique qu'environnemental. L'analyse des nombreux résultats d'essais réalisés sur des sédiments portuaires différents a permis d'étudier la faisabilité technique de ce type de traitement, d'optimiser les dosages et les additifs à mettre en œuvre, et de confirmer la faisabilité environnementale par rapport aux filières de valorisation envisageables pour ces déchets. Les possibilités d'emploi d'un tel traitement sont discutées dans cet article. Il est finalement suggéré de discriminer économiquement les traitements effectués en amont qui permettent d'améliorer la qualité du sédiment pré-traité avant stabilisation/solidification par liant hydraulique.

\section{Mots-clés :}

Sédiments marins contaminés - Stabilisation / Solidification - Traitement par liant hydraulique - Résistance à la compression - Projet SEDIMARD 83.

\section{Abstract :}

The management of contaminated dredged sludge poses problems to the harbour authorities. Marine sediments deposits on land are considered as waste 
materials. A way to reuse the dredged sludge as construction material is possible using binders but the presence of contaminants constitutes some difficulties in the process of stabilization/solidification. To bring industrial solutions, an experimental treatment station ETS was built near Toulon to treat contaminated different harbour sediments. This ETS station was supported by the SEDIMARD 83 project (GROSDEMANGE et al, 2008). One of the aims of the project concerns the valorisation of sediments using binders. Feasibility testing is conducted on various pre treated sediments considering mechanical and environmental aspects. The performed tests have been analyzed and focused on the study of the technical feasibility of the stabilization/solidification treatment by cement, the optimization of replacement level of cement and additives in the respect of environmental conditions or rules applied to the valorisation issues. All the results are discussed in this paper and finally, it is suggested to range taking into account the economic criteria the pre treatments applied before the stabilization/solidification by cement and giving the higher characteristics of resistance.

\section{Keywords :}

Contaminated marine sediments - Stabilization / Solidification - Treatment by cement - Unconfined compressive strength - SEDIMARD 83 project.

\section{Introduction}

En présence de sédiments plus ou moins contaminés, la gestion des dragages représente dès lors une problématique économique et technique pour le développement et le maintien des activités portuaires. Face à une situation de blocage de gestion de ces sédiments contaminés qui ne peuvent pas être clapés en mer, des gestionnaires de ports assistés par des bureaux d'études spécialisés ont initié soit des programmes de recherche ou soit des projets orientés vers une logique opérationnelle pour essayer d'identifier des perspectives possibles de solutions d'emploi à terre de ce nouveau déchet que constitue les sédiments marins contaminés. Dans cette logique, le Conseil Général du Var a initié en 2002 une approche opérationnelle multi partenariale dans le cadre d'un programme spécifique appelé SEDIMARD 83 (GROSDEMANGE et al, 2008). Dans cette opération, un des principaux objectifs était d'effectuer des essais de traitements par inertage des contaminants contenus dans les sédiments acheminés sur le site de l'opération en formant des mélanges à base de liants hydrauliques. Ainsi, de multiples essais ont été réalisés sur les sédiments provenant de différents ports pour vérifier la faisabilité technique et environnementale des procédés d'inertage à base de ciment comme liant hydraulique principal (BOUTOUIL, 1998). 
L'ensemble des essais réalisés a permis de confirmer la faisabilité technique de ce type de traitement; d'identifier les pourcentages optimaux de ciment et les éventuels additifs intéressants à mettre en œuvre; de confirmer la faisabilité environnementale par rapport aux filières de valorisation envisageables; de définir les coupures granulométriques appropriées; et de discriminer économiquement les traitements effectués en amont permettant d'améliorer la qualité du procédé par liant hydraulique.

L'utilisation de la méthode de stabilisation/solidification par traitement à base de liant hydraulique sur les sédiments contaminés doit comprendre trois aspects, à savoir l'un technique et l'autre environnemental, complétée impérativement par une approche économique.

\section{Etude expérimentale}

\subsection{Objet de l'étude pilote SEDIMARD 83}

Cette étude a été élaborée dans le cadre d'un programme de recherche et développement dénommée SEDIMARD 83 visant à répondre aux difficultés de gestion des sédiments portuaires de l'ensemble des maîtres d'ouvrage. Le Conseil Général du Var s'est ainsi positionné comme fédérateur pour la recherche de solutions communes. Ce travail expérimental est destiné à tester la faisabilité technique et environnementale de traitements dans le but d'une stabilisation des contaminants présents dans les sédiments marins. Ces procédés conduisent à obtenir un matériau contrôlé qui pourra être revalorisé dans certains domaines du génie civil, agronomique ou encore en tant que granulats pour matériau de construction.

L'origine des sédiments dragués provient de dix sites indépendants mais complémentaires afin d'être représentatif de la variabilité de ce déchet et de tester la robustesse de chaque procédé de valorisation.

\subsection{Liste des prétraitements effectués}

Les dix sédiments portuaires prélevés dans le cadre de ce pilote ont fait l'objet de divers traitements préalables à l'ajout de liant et ce, individuellement. Ainsi après réception sur le site de l'opération, il est ensuite réalisé les opérations de pré-traitement qui sont au nombre de 8 . Lors de l'arrivée sur le site, les sédiments subissent un tri et une coupure granulométrique et de facto, il se constitue deux catégories de sédiments: bruts (tri des macro-déchets par tamis à 5mm) et dessablés (tri identique au précédent plus hydrocyclonage à $63 \mu \mathrm{m}$ ) qui contiennent les particules les plus fines. Les autres opérations s'appliquent ensuite donc à ces deux catégories à savoir soit une déshydratation qui s'effectue par filtre pressage mécanique; un compostage de quatre mois; une phosphatation 
caractérisée par ajout d'acide phosphorique (environ 5\%); un chaulage qui se constitue d'un ajout de $20 \%$ de chaux vive; une correction granulométrique par apport d'un matériau de carrière (sable) de même granulométrie; ici le pourcentage de sable était de 70 et $85 \%$ pour respectivement 30 et $15 \%$ de sédiment; ou une calcination à $450^{\circ} \mathrm{C}$.

Le tableau 1 regroupe les différentes chaînes de traitement que le sédiment a subi (pré-traitement) et leurs numéros d'échantillon. Dans le cadre des travaux de recherche, les sédiments présentés ci-dessous sont tous caractérisés chimiquement avant incorporation de ciment. Ceux-ci ont montré une grande diversité des formes chimiques sous lesquelles se trouvent les métaux. Cependant, il n'est pas exclu que des comportements similaires puissent exister lors d'un traitement par liant.

Tableau 1 : Liste des procédés de traitements et leurs abréviations.

\begin{tabular}{|c|l|c|}
\hline Abréviation & \multicolumn{1}{|c|}{ Chronologie des traitements } & $\begin{array}{c}\text { Echantillon } \\
\text { Numéro }\end{array}$ \\
\hline DDCA & DESSABLE - DESHYDRATE - CALCINE 450 ${ }^{\circ} \mathrm{C}$ & 2 \\
\hline DD & DESSABLE - DESHYDRATE & 6 \\
\hline DCO & DESSABLE - COMPOSTE & 11 \\
\hline BCA & BRUT- CALCINE 450 ${ }^{\circ}$ C & 15 \\
\hline BCO & BRUT- COMPOSTE & 19 \\
\hline B & BRUT & 21 \\
\hline BPCO & BRUT- PHOSPHATE - COMPOSTE & 24 \\
\hline DCH & DESSABLE - CHAULE & 27 \\
\hline BCH & BRUT-CHAULE & 29 \\
\hline BCO-PD & BRUT- COMPOSTE - PHOSPHATE- DESHYDRATE & 34 \\
\hline $\begin{array}{c}\text { BCO-CG } \\
\text { (MA) }\end{array}$ & $\begin{array}{l}\text { BRUT- COMPOSTE - CORRECTION GRANULOMETRIQUE } \\
\text { (MATERIAU ALTERNATIF) }\end{array}$ & 35 \\
\hline $\begin{array}{c}\text { BCO-PD-CG } \\
\text { (MA) }\end{array}$ & BRUT- COMPOST- PHOSPHATE - DESHYDRATE - CORRECTION \\
\hline GRANULOMETRIQUE (MATERIAU ALTERNATIF) & 36 \\
\hline $\begin{array}{c}\text { DCO-P } \\
\text { (MA) }\end{array}$ & DESSABLE - COMPOSTE - PHOSPHATE & $\begin{array}{l}\text { BRUT-PHOSPHATE -COMPOSTE - CORRECTION } \\
\text { GRANULOMETRIQUE (MATERIAU ALTERNATIF) }\end{array}$ \\
\hline DPCO & DESSABLE - PHOSPHATE - COMPOSTE & 41 \\
\hline
\end{tabular}

\subsection{Méthodes}

Le traitement des sédiments par liant hydraulique est préparé suivant deux mélanges : mélanges "sédiment-ciment" et mélanges "sédiment-ciment-additif". Pour ces dernières formulations, l'objectif est d'étudier l'influence de la présence d'une certaine quantité d'additif réagissant avec les constituants du sédiment.

Les constituants des mélanges sont introduits dans le malaxeur dans l'ordre : vase, additif s'il y a lieu, puis ciment de façon à respecter la cinétique des réactions en présence. Le ciment utilisé est un ciment de type CEM I PM de classe 52,5 
$(\mathrm{CaO}=65,3 \%)$. Les additifs sont au nombre de deux et peuvent être soit de la chaux vive $(\mathrm{CaO}=92 \%)$ soit de la soproline ${ }^{\circledR}$ (cendre volante à $32.3 \%$ de $\left.\mathrm{CaO}\right)$, voir tableau 2.

Les proportions envisagées tiennent compte de deux composantes: une composante économique limitant la quantité de liant utilisé, et une mécanique liée à sa résistance à la compression simple (ici, le seuil de référence est fixé à $1 \mathrm{MPa}$ ) qui est fonction de la proportion de ciment. Une fois la mise en œuvre terminée, le matériau est incorporé dans des moules cylindriques de diamètre $6 \mathrm{~cm}$ et de hauteur $12 \mathrm{~cm}$. La durée de stockage à température ambiante est de 28 jours.

Tableau 2 : Formulations testées sur les sédiments marins

\begin{tabular}{|c|c|c|c|c|c|}
\hline Formulation & Sédiment sec & CEM I & Soproline & Chaux vive & Note économique \\
\hline A1 & 100 & 5 & 0 & 0 & 1.0 \\
\hline A2 & 100 & 10 & 0 & 0 & 2.0 \\
\hline A3 & 100 & 12.5 & 0 & 0 & 2.5 \\
\hline A4 & 100 & 15 & 0 & 0 & 3.0 \\
\hline B1 & 100 & 15 & 0 & 10 & 4.0 \\
\hline B2 & 100 & 10 & 20 & 10 & 3.4 \\
\hline B3 & 100 & 10 & 0 & 5 & 2.5 \\
\hline B4 & 100 & 10 & 20 & 5 & 2.9 \\
\hline B5 & 100 & 20 & 0 & 10 & 5.0 \\
\hline B6 & 100 & 15 & 30 & 10 & 4.6 \\
\hline B7 & 100 & 15 & 35 & 15 & 5.2 \\
\hline B8 & 100 & 15 & 0 & 5 & 3.5 \\
\hline
\end{tabular}

Nota : Les chiffres indiquent la masse de chaque constituant en $\mathrm{g}$ pour $100 \mathrm{~g}$ de sédiment sec

\section{Approche discriminatoire économique}

L'idée de cette approche est de tenter de discriminer des pré-traitements ou procédés de traitements qui permettent à des moindres coûts de préparer les sédiments aux traitements à base de liant hydraulique et de répondre au double objectif de la méthode : solidification et stabilisation. L'indicateur pour mesurer la qualité au comportement mécanique des sédiments solidifiés est la résistance à la compression simple à 28 jours sur des éprouvettes cylindriques d'élancement 2. La stabilisation est quant-à elle associée au comportement environnemental évalué à partir des essais de lixiviation (mises en solution) sur monolithe (NF X 31-211, 2000).

A l'issue des résultats, nous avons affecté une note «économique » sur les matériaux obtenus après incorporation de liant hydraulique quels que soient le ou les pré-traitements subis. Cette note globale résulte de la somme de deux notes, à savoir : 
- une note en fonction de la chaîne de pré-traitement et/ou traitement appliquée avant traitement par liant hydraulique qui varie de 0 (sédiment brut) à 7 (sédiment dessablé déshydraté calciné). Cette pondération correspond à l'addition de note affectée individuellement à chaque procédé. Elle est établie en fonction de la faisabilité technique et des moyens nécessaires à sa mise en œuvre (humains, matériels et consommables) et est synthétisée dans le tableau 3.

Tableau 3 : Pondération des traitements réalisés sur le projet SEDIMARD 83.

\begin{tabular}{|c|c|c|c|c|c|c|c|c|c|c|}
\hline Procédé & Brut & Criblage & Cor. Gran. & Dessablé & \begin{tabular}{|l|} 
Deshydraté \\
\end{tabular} & COmposté & Phosphaté & CHaulé & CAlciné & \multirow[b]{3}{*}{ Note traitement } \\
\hline Note procédé & 0.0 & 0.5 & 0.5 & 0.5 & 1.0 & 1.5 & 1.5 & 2.0 & 5.5 & \\
\hline & & & & & & & & & & \\
\hline B & $\bar{X}$ & & & & & & & & & 0.0 \\
\hline $\mathrm{DD}$ & $\bar{X}$ & & & $\bar{X}$ & $\bar{X}$ & & & & & 1.5 \\
\hline $\mathrm{BCO}$ & $\bar{X}$ & & & & & $\bar{X}$ & & & & 1.5 \\
\hline$\overline{\mathrm{DCO}}$ & $\bar{X}$ & & & $\bar{X}$ & & $\bar{X}$ & & & & 2.0 \\
\hline $\mathrm{BCH}$ & $\bar{X}$ & & & & & & & $\bar{X}$ & & 2.0 \\
\hline BCO-CG (MA) & $\bar{X}$ & & $\bar{X}$ & & & $\bar{X}$ & & & & 2.0 \\
\hline$\overline{\mathrm{DCH}}$ & $\bar{X}$ & & & $\bar{X}$ & & & & $\bar{X}$ & & 2.5 \\
\hline $\mathrm{BPCO}$ & $\bar{X}$ & & & & & $\bar{X}$ & $\bar{X}$ & & & 3.0 \\
\hline DCO-P & $\bar{X}$ & & & $\bar{X}$ & & $\bar{X}$ & $\bar{X}$ & & & 3.5 \\
\hline BPCO.CG (MA) & $\bar{X}$ & & $\bar{X}$ & & & $\bar{X}$ & $\bar{X}$ & & & 3.5 \\
\hline $\mathrm{DPCO}$ & $\bar{X}$ & & & $\bar{X}$ & & $\bar{X}$ & $\bar{X}$ & & & 3.5 \\
\hline BCO.PD & $\bar{X}$ & $\bar{X}$ & & & $\bar{X}$ & $\bar{X}$ & $\bar{X}$ & & & 4.5 \\
\hline$\overline{B C O-P D . C G ~(M A) ~}$ & $\bar{X}$ & $\bar{X}$ & $\bar{X}$ & & $\bar{X}$ & $\bar{X}$ & $\bar{X}$ & & & 5.0 \\
\hline$\overline{B C A}$ & $\bar{X}$ & $\bar{X}$ & & & & & & & $\bar{X}$ & 6.0 \\
\hline DDCA & $\bar{X}$ & & & $\bar{X}$ & $\bar{X}$ & & & & $\bar{X}$ & 7.0 \\
\hline
\end{tabular}

- une note sur les formulations mises en œuvre, établie à partir des dosages en ciment employé en prenant pour principe que plus la note est élevée, plus la quantité de ciment et d'additif mise en œuvre est importante.

Ainsi une notation globale élevée signifie que le procédé est onéreux.

Les notes finales obtenues, pour chacun des traitements répondant positivement aux tests environnementaux définissant la notion d'un matériau valorisable en techniques routières (GTS, 2000) d'après les seuils d'acceptabilité en remblai revêtu (CCTP, 2006), sont regroupées dans le tableau 4. Elles sont couplées aux résultats mécaniques obtenus, à savoir à la valeur de la résistance à la compression simple acquise à 28 jours (notée $\mathrm{Rc}_{28}$ ).

Tableau 4 : Notes finales obtenues pour chacun des traitements - SEDIMARD 83.

\begin{tabular}{|c|c|c|c|c|c|}
\hline Abréviation & Formulation & Note $^{*}$ & Note $^{* *}$ & Note globale & $R c_{28} \mathrm{MPa}$ \\
\hline BCH & A4 & 2.0 & 3.0 & 5.0 & 1.14 \\
\hline \hline BCO & A2 & 1.5 & 2.0 & 3.5 & 1.01 \\
\hline BCO & A3 & 1.5 & 2.5 & 4.0 & 1.08 \\
\hline BCO & A4 & 1.5 & 3.0 & 4.5 & 1.56 \\
\hline BCO & A4 & 1.5 & 3.0 & 4.5 & 1.05 \\
\hline BCO & B1 & 1.5 & 4.0 & 5.5 & 0.82 \\
\hline BCO & B2 & 1.5 & 3.4 & 4.9 & 1.29 \\
\hline BCO & B8 & 1.5 & 3.5 & 5.0 & 1.18 \\
\hline
\end{tabular}




\begin{tabular}{|c|c|c|c|c|c|}
\hline $\mathrm{BCO}$ & B4 & 1.5 & 2.9 & 4.4 & 1.33 \\
\hline BCO-CG (M.A.) & B3 & 2.0 & 2.5 & 4.5 & 3.25 \\
\hline BCO-CG (M.A.) & B4 & 2.0 & 2.9 & 4.9 & 9.00 \\
\hline $\mathrm{DCH}$ & A4 & 2.5 & 3.0 & 5.5 & 1.30 \\
\hline DCO & B5 & 2.0 & 5.0 & 7.0 & 1.33 \\
\hline DCO & B6 & 2.0 & 4.6 & 6.6 & 1.39 \\
\hline DCO & B7 & 2.0 & 5.2 & 7.2 & 1.57 \\
\hline BPCO & A4 & 3.0 & 3.0 & 6.0 & 2.07 \\
\hline BPCO & B1 & 3.0 & 4.0 & 7.0 & 1.61 \\
\hline BPCO & B2 & 3.0 & 3.4 & 6.4 & 2.50 \\
\hline DPCO & A4 & 3.5 & 3.0 & 6.5 & 2.01 \\
\hline DPCO & B1 & 3.5 & 4.0 & 7.5 & 1.61 \\
\hline DPCO & B2 & 3.5 & 3.4 & 6.9 & 2.35 \\
\hline BCA & A1 & 6.0 & 1.0 & 7.0 & 1.37 \\
\hline BCA & A2 & 6.0 & 2.0 & 8.0 & 2.61 \\
\hline $\mathrm{BCA}$ & A4 & 6.0 & 3.0 & 9.0 & 3.74 \\
\hline DDCA & A1 & 7.0 & 1.0 & 8.0 & 1.76 \\
\hline DDCA & A2 & 7.0 & 2.0 & 9.0 & 2.99 \\
\hline DDCA & A4 & 7.0 & 3.0 & 10.0 & 3.66 \\
\hline DCO-P & B5 & 3.5 & 5.0 & 8.5 & 1.40 \\
\hline DCO-P & B6 & 3.5 & 4.6 & 8.1 & 1.91 \\
\hline DCO-P & B7 & 3.5 & 5.2 & 8.7 & 2.01 \\
\hline BCO-PD & A4 & 4.5 & 3.0 & 7.5 & 0.90 \\
\hline BCO-PD & B1 & 4.5 & 4.0 & 8.5 & 0.84 \\
\hline BCO-PD & B2 & 4.5 & 3.4 & 7.9 & 1.22 \\
\hline BCO-PD-CG (M.A) & B3 & 5.0 & 2.5 & 7.5 & 3.75 \\
\hline BCO-PD-CG (M.A) & B4 & 5.0 & 2.9 & 7.9 & 9.50 \\
\hline
\end{tabular}

Nota : * note relative au traitement; **note relative à la formulation en liant.

\section{Les séries des mélanges}

Une première série de mélanges $(\mathrm{A})$ a été préparée avec des teneurs en ciment de $0.5,1,1.25$ et $1.5 \mathrm{~kg}$ pour $10 \mathrm{~kg}$ de sédiment sec. Dans une deuxième série $(\mathrm{B})$, on a incorporé sur les additifs avec des pourcentages de 5, 10 et $15 \%$ de ciment. Pour les liants, une note de 1 est affectée pour les formulations contenant $5 \%$ de ciment, une note de 0.5 pour $5 \%$ d'incorporation de chaux et une note de 0.1 est affectée pour la soproline ajoutée à $5 \%$. Les quantités et les pondérations de tous les mélanges étudiés sont répertoriées dans le tableau 2.

Les valeurs des résistances mécaniques sont relativement faibles du fait de la nature des matériaux stabilisés, voir tableau 4. En effet, les matrices traitées représentent majoritairement des fines riches en matières organiques qui ne présentent pas de squelette minéral capable de supporter des contraintes verticales et où le liant est sensé apporter la cohésion à ce squelette. 


\section{Visualisation et analyse des évaluations}

Afin de discriminer les différents traitements au regard du volet économique, une visualisation des résultats obtenus est proposée sous forme de deux graphiques correspondant aux objectifs visés dans le projet SEDIMARD 83.

- Figure 1 - L'obtention d'une valeur de $1 \mathrm{MPa}$ (valeur seuil minimale) en résistance à la compression simple faisait partie de l'objectif visé pour le volet comportement mécanique. Ceci se traduit par l'étude comparative des valeurs en résistance obtenues à 28 jours pour l'ensemble des chaînes de traitement.

- Figure 2 - L'apport du correcteur granulométrique améliore le squelette granulaire et donne des résistances nettement plus élevées. Ce type de traitement correspond à la mise au point d'un matériau alternatif. Dans la figure 2 , on ne considère plus de type de matériau alternatif et ainsi on retrouve les valeurs regroupées entre 1 et $4 \mathrm{MPa}$.

Tableau 5 - Symboles utilisés - figures 1 et 2

\begin{tabular}{|c|c|c|c|c|}
\hline$\diamond \mathrm{B}$ & $\mathrm{BCH}$ & $\mathrm{OBCO}$ & + BCO-CG (M.A.) & $\Delta \mathrm{DD}$ \\
\hline$\times D C H$ & $\square D C O$ & $\triangle \mathrm{BPCO}$ & $+B P C O-C G(M . A)$ & $\bullet \mathrm{DPCO}$ \\
\hline$O B C A$ & * DDCA & DCO-P & $\bullet \mathrm{BCO}-\mathrm{PD}$ & + BCO-PD-CG (M.A) \\
\hline
\end{tabular}

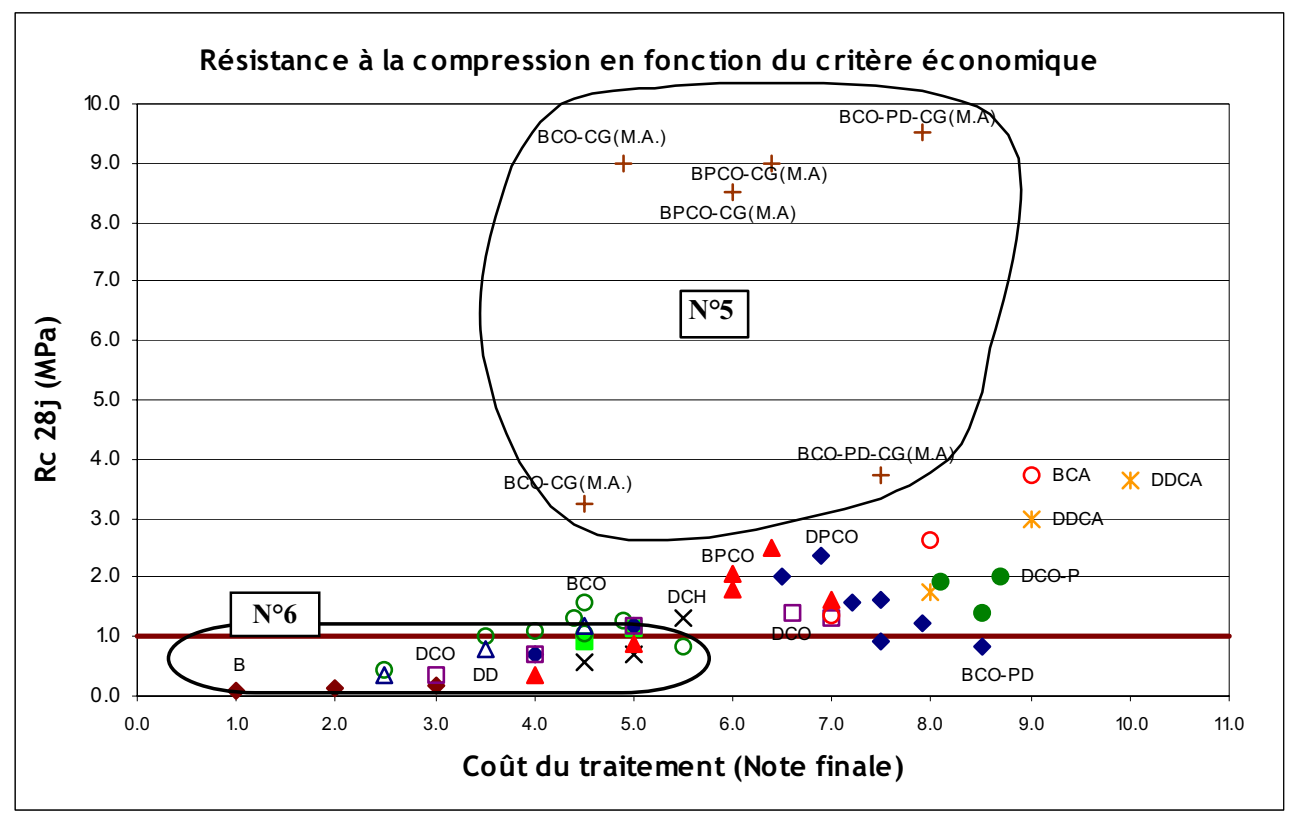

Figure 1 - Relation $R c_{28}$ en fonction du coût du traitement global 


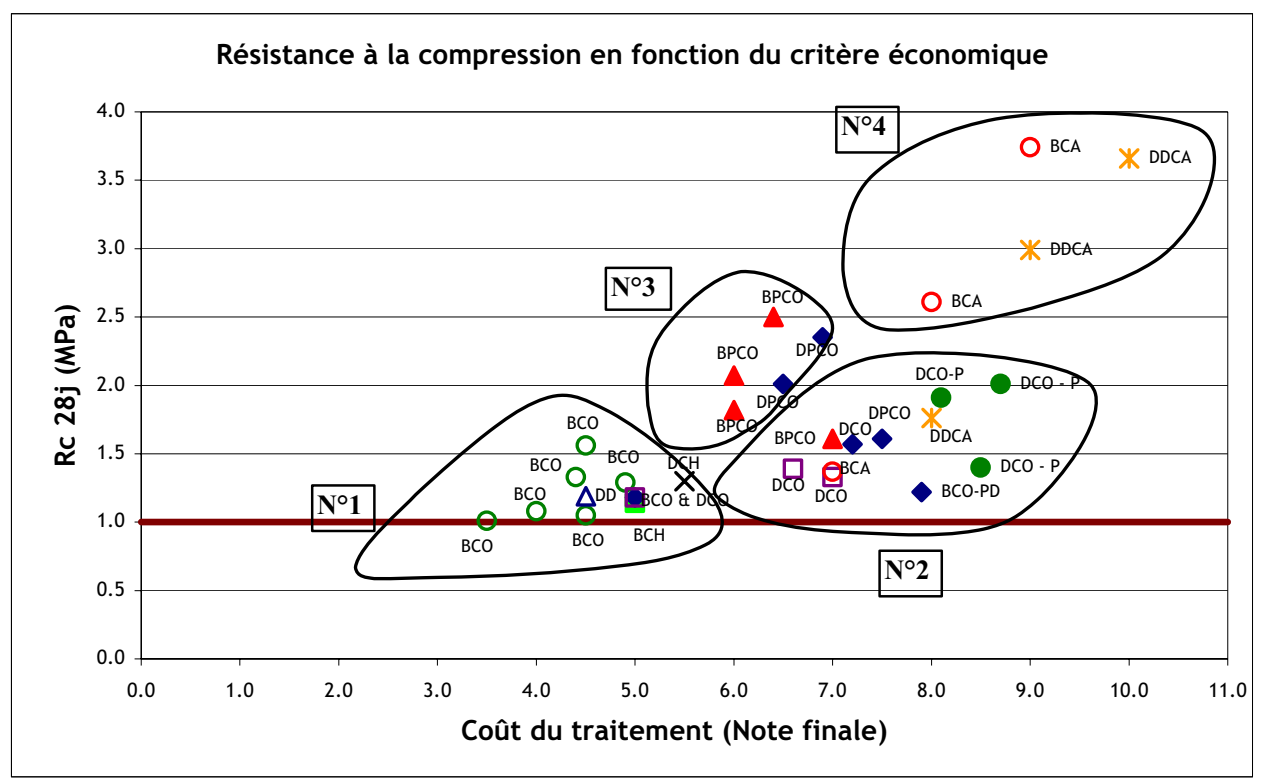

Figure 2 - Relation $R c_{28}$ en fonction du coût du traitement global (supérieur à 1 MPa et sans apport granulaire)

Des tendances observées et identifiées sur les figures se dégagent six groupes de traitements et/ou familles de sédiments. On a donc, avec entre parenthèses la moyenne des traitements :

- Groupe $\mathrm{n}^{\circ} 1$ (coût $=4.6 ; \mathrm{Rc}_{28} 51.2 \mathrm{MPa}$ ) : Il comprend les sédiments traités par compostage sur le brut et par chaulage sur les deux granulométries. Le procédé est économiquement faible mais les résistances ne sont pas nettement au-dessus du seuil.

- Groupe $\mathrm{n}^{\circ} 2$ (coût $=7.6 ; \mathrm{Rc}_{28} 51.55 \mathrm{MPa}$ ): Il concerne les sédiments compostés suivis d'une phosphatation et ceux dessablés compostés. Le bénéfice sur le comportement mécanique est très faible au vu du traitement dispendieux.

- Groupe $\mathrm{n}^{\circ} 3$ (coût $=6.3 ; \mathrm{Rc}_{28} 52.1 \mathrm{MPa}$ ): Il prend en compte tous les matériaux phosphatés suivis d'un compostage. On trouve ici un bon compromis entre les performances mécaniques et le facteur économique.

- Groupe $\mathrm{n}^{\circ} 4$ (coût $=9.0 ; \mathrm{Rc}_{28} 53.3 \mathrm{MPa}$ ) : Il est régi par le procédé de calcination. Le bénéfice sur le comportement mécanique est certes important mais il s'accompagne par un coût prohibitif.

- Groupe ${ }^{\circ} 5$ (coût $\left.=6.2 ; \mathrm{Rc}_{28} 57.2 \mathrm{MPa}\right)$ : Il comprend l'ensemble des matériaux dits alternatifs. Il semble que ce mélange est le plus intéressant. Cependant, la contrepartie est la faible quantité de sédiment traité.

- Groupe $n^{\circ} 6$ (coût $=4.0 ; \mathrm{Rc}_{28} 50.55 \mathrm{MPa}$ ): Comme l'on pouvait s'y attendre, les éprouvettes contenant les plus faibles pourcentages de ciment 
et sans additif présentent les plus faibles compressions. Cela concerne plus particulièrement tous les sédiments bruts et dessablés déshydratés. Ces sédiments ne sont pas appropriés à ce type de mélange.

\section{Conclusions}

L'étude, présentée dans cette communication, a mis en œuvre une méthodologie de caractérisation chimique, physique et économique qui permet d'identifier les procédés les plus efficaces à un traitement par liants hydrauliques. Elle a consisté à montrer que la solidification et la stabilisation obtenues peuvent être suffisantes dans le cas d'une utilisation dans certains domaines de la construction où de grandes résistances ne sont pas nécessaires. Ainsi, d'après les premières investigations, les conclusions suivantes peuvent être énoncées :

- Le procédé de phosphation accompagné d'un compostage apporte le meilleur compromis résistance/coût pour un traitement au liant hydraulique.

- L'ajout d'additif, moins cher, à ce traitement est bénéfique notamment parce qu'ils sont consommateur d'eau et peuvent détruire la matière organique.

- Une solution alternative à une calcination ou une phosphatation onéreuse est l'emploi de plus faibles pourcentages de sédiments marins en complétant par un matériau de carrière. En effet, par son apport d'éléments grossiers, il réduit les concentrations en agents inhibiteurs à la prise du liant et augmente la cohésion du squelette minéral.

\section{Références bibliographiques}

GROSDEMANGE D., LEVEQUE F., DROUSIE D., AQUA J.L., MEHU J., BAZIN C. (2008). The SEDIMARD project: presentation and results. International Symposium on Sédiment Management-I2SM, Lille, pp 181-186. CCTP (2006). Acceptabilité de déchets et de matériaux en techniques routières Edition MTETM-MEDD-ADEME-BRGM-CETE-INERIS-INSA-LCPC-SETRA, pp $66 / 82$.

GTS (2000). Traitement des sols à la chaux et aux liants hydrauliques - Guide technique - Edition LCPC-SETRA.

BOUTOUIL M. (1998). Traitement des vases de dragage par solidification /stabilisation à base de ciments et additifs - Thèse de doctorat, Université du Havre, $276 \mathrm{p}$.

REY F. (1998). Etude physico-mécanique et environnementale du composite vase-chaux et additifs - Thèse de doctorat, Université du Caen, $277 \mathrm{p}$.

NF X 31- 211 (2000). Déchets - Essais de lixiviation d'un déchet solide initialement massif ou généré par un procédé de solidification. 